\title{
Index rerum ad vol. 23
}

Abcès scrotaux simulants une hernie inguinale, 351 Autotransplantation rénale, 224

Blasenersatzoperationen, 436 Blasenkarzinom, transurethrale Resektion, 244 Blasenscheidenfisteln nach Hysterekto-

mie, 230 Bricker Operation, 89

Calculos coraliformes, el colgajo pielorenal, 326 Calculs d'acid urique, 411

- $\quad$ du rein, dissolution chimique, 236Cancer de la vessie, resection transurethrale, 244

- $\quad$ de Touraque, clinique et traitement,519

Carcinoma of the bladder, transurethral

resection, 244 Chemolysis of renal lithiasis, 236 Cowperitis calculosa, 549

Derivation urinaire artificielle chez

Tenfant, 1 -, indications, 8

-, dans le sigmoide non isolé, 15 -, dans le colon isolé, 53 -, dans Гiléon isolé, 82 -,

implantations cutanées, 103 -, réintervention, 135 Duplication of bladder, urethra and

lower genital organs, 514

Estenosis de la uretra posterior, invagi-

nación, 364 Ethyl Carbamate, renal response, 309 Extrophic bladder, reconstruction in one session, 151

Fistules vaginales après hystérectomie, 230

Genitaltuberkulose des Mannes und

chemisches Milieu, 356 Gersuny Operation, 75

Haematuria, 275 Harnableitung beim Kinde, 1 -, Indikationen, 8

in den ausgeschalteten Dickdarm, 53

in den ausgeschalteten Dünndarm, 82

in den nicht ausgeschalteten Dickdarm, 15

in die Haut, 103

-, Revision bei Mängeln, 135

Harnblase, Aufbau bei Exstrophie, 151

Harnleukozyten bei entzündlichen Er-

krankungen, 377

Harnsäuresteinbildung, 411

Heitz-Boyer-Hovelacque Operation, 63, 74

Hirschgeweihsteine, eine neue Entfer-nungstechnik, 326

Histerectomia, fistulas vesico-vaginales, 230

Homotransplantation rénale, nouvelle technique, 201

Hyperparathyredoisismus et urolithiasis, 315

Hypertension rénovasculaire, autotransplantation, 224

Incontinence d'effort dans le prolapsus 
genital, 561 Intracapsular prostatic resection, 535

Leucocytes urinaires dans les pyéloné-

phrites, 377 Litogénesis urica, 411

Maydl Operation, 41

Missbildung von Blase, Harnröhre und

Genitalorganen, 514 Moniliasis albicans vesicae, 428 Muguet de la vessie, 428

Index rerum

Nephro-seminal vesicular malformation,

492 Nierenautotransplantation, 224 Nierenhomotransplantation, neue Tech-

nik, 201 Nieren-Samenblasen-Missbildung, 492 Nierensteinchemolyse, instrumentelle,

236 Nierenteilresektion und Hypoxieresi-

stenz, 388 Nierentuberkulose, Radioisotopen-Dia-

gnostik, 263

Partial renal resection and resistance to hypoxia, 388

Prolapso genital, incontinencia de stress, 561

Prostataresektion, intrakapsuläre, 535

Prostatectomia suprapubica y la resection transuretral, estudion compara-tivo, 454

Prostatectomie transvésicale sans sonde, 447

Pyelo-interstitieller Reflux, 289

Pyuria in infants and young children, 335

Reflux pyélo-interstitiel, 289

Renal autotransplantation, 224

-, homotransplantation, new surgical technique, 201

-, tuberculosis, radioisotopes in diagnosis, 263

Renolitoquimiolisis, 236

Resection prostatique intracapsulaire, 535

Reno vascular hypertension, autotransplantation, 224

Riñón, auto trasplante, 224

-, homotrasplante, nueva técnica, 201

Scrotal abscesses simulating inguinal

hernias, 351 Staghorn calculi, a new technique of

removal, 326 Sternheimer-Malbin cells, 377 Stressinkontinenz bei Genitalprolaps,

561

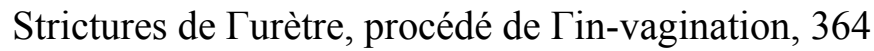

Strikturen der hinteren Harnröhre, Invaginationsverfahren, 364

Suprapubische Prostatektomie und transurethrale Elektroresektion, Spät-ergebnisse, 454

Supravesical urinary diversion in children, 130

Tiersch-Tuffier Operation, 49 Transvesikale Prostatektomie ohne Ka-

theter, 447 Tuberculose rénale, le diagnostic isoto-

pique, 263 Tuberculosis of the male genital organs,

356

Urachuskarzinom, Klinik und Therapie,

519 Uretero-sigmoidostomia, 15 Ureterostomia cutanea, 103 Urethral stricture, invagination technic,

364 Urethane, renal response, 309 Uric acid calculi, 411 Urinary Diversion in children, 1 by cutaneous ureterostomy, 103 
by isolated ileal loop, 82 -, indication, 8

in the not excluded great gut, 15

- $\quad$ into an excluded part of the largeintestine, 53

-, revision, 135

- $\quad$ leucocytes in inflammatory diseases, 377

Urolithiasis et hyperparathyreoidismus, 315

Vejiga, resección transuretral del carcinoma, 244

-, sustituciones, 436

-, urinaria, monilias, 428

Vesical prothesis, 436

Vesico-vaginal fistulas after hysterectomy, 230

$\mathrm{V}$. Vessie extrophique, reconstruction en un temps, 151

-, les remplacements, 436 